\title{
No inferioridad entre dos agentes de bajo volumen (picosulfato de sodio/citrato de magnesio frente al sulfato de sodio/potasio/magnesio) en la preparación del colon para procedimientos diagnósticos: estudio observacional
}

\author{
Non-inferiority between two low-volume agents (sodium picosulfate/magnesium \\ citrate vs. sodium sulfate/potassium/magnesium) to prepare the bowel for \\ diagnostic procedures: an observational study
}

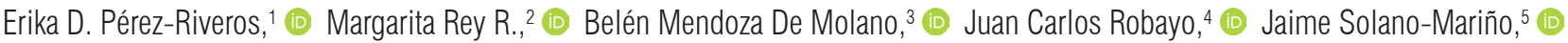

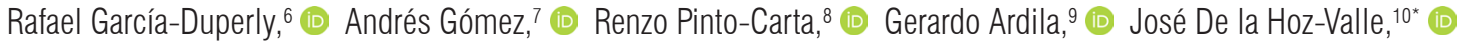 \\ Fernando Sierra-Arango. ${ }^{11}$ (D)
}

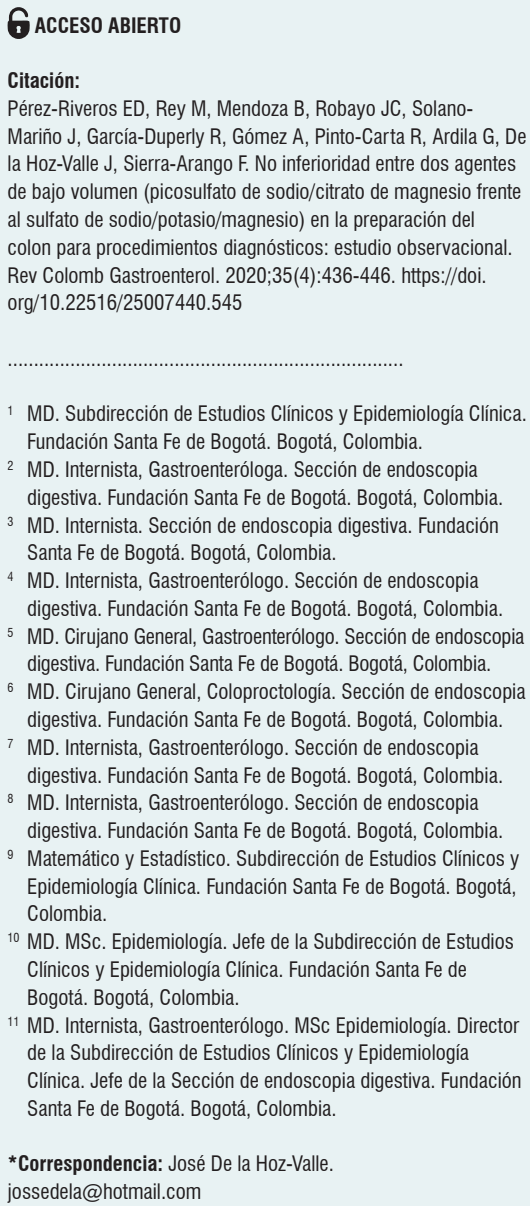

MD. Subdirección de Estudios Clínicos y Epidemiología Clínica. Fundación Santa Fe de Bogotá. Bogotá, Colombia.

MD. Internista, Gastroenteróloga. Sección de endoscopia digestiva. Fundación Santa Fe de Bogotá. Bogotá, Colombia MD. Internista. Sección de endoscopia digestiva. Fundación Santa Fe de Bogotá. Bogotá, Colombia.

MD. Internista, Gastroenterólogo. Sección de endoscopia digestiva. Fundación Santa Fe de Bogotá. Bogotá, Colombia. MD. Cirujano General, Gastroenterólogo. Sección de endoscopia digestiva. Fundación Santa Fe de Bogotá. Bogotá, Colombia.

MD. Cirujano General, Coloproctología. Sección de endoscopia digestiva. Fundación Santa Fe de Bogotá. Bogotá, Colombia. MD. Internista, Gastroenterólogo. Sección de endoscopia digestiva. Fundación Santa Fe de Bogotá. Bogotá, Colombia. MD. Internista, Gastroenterólogo. Sección de endoscopia digestiva. Fundación Santa Fe de Bogotá. Bogotá, Colombia. Matemático y Estadístico. Subdirección de Estudios Clínicos y Epidemiología Clínica. Fundación Santa Fe de Bogotá. Bogotá, Colombia.

${ }^{10}$ MD. MSc. Epidemiología. Jefe de la Subdirección de Estudios Clínicos y Epidemiología Clínica. Fundación Santa Fe de Bogotá. Bogotá, Colombia.

MD. Internista, Gastroenterólogo. MSc Epidemiología. Director de la Subdirección de Estudios Clínicos y Epidemiología

Clínica. Jefe de la Sección de endoscopia digestiva. Fundación Santa Fe de Bogotá. Bogotá, Colombia

*Correspondencia: José De la Hoz-Valle. jossedela@hotmail.com

Fecha recibido: $23 / 04 / 20$

Fecha aceptado: 05/10/20

\section{Resumen}

Introducción: el cáncer colorrectal es un problema de salud pública; sin embargo, la detección temprana reduce su morbimortalidad. La colonoscopia es el procedimiento de elección para detectar lesiones premalignas y el éxito depende de una limpieza adecuada. El objetivo es evaluar el desempeño de dos preparaciones de bajo volumen empleados en un hospital de alto nivel. Materiales y métodos: estudio prospectivo en adultos que asistieran a colonoscopia en la Fundación Santa Fe de Bogotá, Colombia. Las preparaciones se evaluaron con la escala de Boston, con puntaje $\geq 6$ puntos para una limpieza adecuada. Se realizó un análisis de regresión logística para establecer la efectividad de los medicamentos con un cálculo de no inferioridad del $3 \%-5 \%$. Resultados: 598 pacientes fueron evaluados. El $49 \%$ (293) fue expuesto al picosulfato de sodio/citrato de magnesio y el $51 \%$ (305) fue expuesto al sulfato de sodio/potasio/magnesio. Con un promedio de Boston de 6,98 $\pm 1,86$ (78 \% con puntaje de Boston $\geq 6)$ y $7,39 \pm 1,83(83 \%)$, respectivamente $(p=0,649)$. Según el análisis de la presencia y frecuencia de síntomas no deseados, el picosulfato fue mejor tolerado $(p<0,001)$. Conclusiones: los estudios de preparación intestinal en pacientes de un escenario real son muy escasos. Los medicamentos de bajo volumen obtuvieron una efectividad global y por segmento de colon similar, confirmando la no-inferioridad; el picosulfato de sodio/citrato de magnesio fue mejor tolerado. Un estudio de costo-efectividad podría definir esto según las necesidades de la población de estudio.

\section{Palabras clave}

Neoplasias colorrectales, picosulfato de sodio, preparación intestinal, evidencia en la vida real.

\begin{abstract}
Introduction: Colorectal cancer is a public health problem; however, early detection reduces morbidity and mortality. Colonoscopy is the procedure of choice for detecting precancerous lesions, and success depends on proper bowel cleansing. Objective: To evaluate the performance of two low-volume agents used in a high-level hospital. Materials and methods: Prospective study in adults who underwent colonoscopy at the Fundación Santa Fe in Bogotá, Colombia. Preparations were evaluated using the Boston Bowel Preparation Scale. A score $\geq 6$ points indicated adequate preparation. A logistic regression analysis was carried out to establish the effectiveness of the medicines with a non-inferiority ratio of 3-5\%. Results: 598 patients were evaluated. $49 \%$ (293) received sodium picosulfate/magnesium citrate and $51 \%$ (305) received sodium sulfate/potassium/magnesium, with an average Boston score of $6.98 \pm 1.86(78 \%$ Boston $\geq 6)$ and $7.39 \pm 1.83(83 \%)$, respectively ( $p=0.649)$. According to the analysis of the presence and frequency of unwanted symptoms, picosulfate was better tolerated $(p<0.001)$. Conclusions: Bowel preparation studies in patients from a real-life scenario are scarce. Low-volume agents had similar overall and segmental effectiveness in the colon, confirming non-inferiority; sodium picosulfate/magnesium citrate was better tolerated. A cost-effectiveness study could establish the best option according to the needs of the study population.
\end{abstract}

Keywords

Colorectal neoplasms, Sodium picosulfate, Intestinal preparation, Real-llife evidence. 


\section{INTRODUCCIÓN}

El cáncer colorrectal (CCR) es un problema de salud pública (1). Según datos de la Organización Mundial de la Salud (OMS), se estimaron más de 1,8 millones de casos nuevos de CCR y 881000 muertes para el 2018 a nivel global (2). En Colombia se estima que 12 de cada 100000 personas se diagnostican con CCR al año (50\% fallece) (3), cifra importante debido al impacto que esto genera para el sistema de salud del país. La detección temprana de CCR reduce su morbimortalidad (4), y la colonoscopia es el procedimiento de elección y el más frecuentemente utilizado (5-7).

Este procedimiento permite la detección de múltiples patologías de íleo terminal y colon (infecciosas, inflamatorias, hemorrágicas y neoplásicas) (8-10). Con respecto al tamizaje de CCR, este permite un manejo inmediato ante las lesiones benignas o premalignas (11). El $90 \%$ de los casos de CCR se diagnostica en personas mayores de 50 años (12), puesto que el tamizaje debe iniciar a partir de esta edad para pacientes de bajo riesgo y a partir de los 40 años para los de alto riesgo (13). El éxito de este procedimiento depende de una preparación apropiada que permite una mejor visualización $(14,15)$. La reprogramación del procedimiento secundario a una preparación inadecuada implica un retraso del diagnóstico de lesiones premalignas hasta en un $46 \%$ de los pacientes $(16,17)$.

Actualmente, existen más de 10 productos disponibles para preparación intestinal a nivel global (18). Múltiples estudios han comparado diferentes métodos y combinaciones de las preparaciones intestinales disponibles $(19,20)$. El polietilenglicol (PEG) es uno de los productos más utilizados debido a su alta eficacia y baja incidencia de alteraciones hidroelectrolíticas, particularmente en pacientes con múltiples comorbilidades $(21,22)$. Sin embargo, presenta una pobre tolerabilidad (requerimiento de alta ingesta de líquidos y baja palatabilidad) determinando una alta probabilidad de preparación incompleta e inadecuada (23).

El picosulfato de sodio/citrato de magnesio (SPMC) es una preparación de bajo volumen que actúa como laxante estimulante y osmótico. La eficacia de este medicamento ha demostrado resultados favorables, incluso superiores en tolerabilidad y seguridad (24); hipótesis contrastada con algunos estudios que evidenciaron un mayor riesgo de alteraciones hidroelectrolíticas $(25,26)$; por esto, se recomienda que los pacientes mayores de 65 años no se preparen con medicamentos hiperosmolares $(27,28)$.

Existen diferentes escalas para determinar la eficacia de la preparación intestinal. La escala de Boston (BBPS) es una escala validada internacionalmente de 9 puntos $(0=\sin$ preparación; 9 = óptima preparación), separando las 3 secciones del colon. Una particularidad presente en esta escala es que la valoración se realiza después de un lavado y succión realizado por el operador $(29,30)$. Cada sección (derecho, trasverso e izquierdo) tiene una puntuación de 0 a 3 ( $0=$ colon sin preparar, no valorable por presencia de residuos fecales sólidos no lavables; 1 = algunas áreas son valorables, residuo fecal; 2 = mucosa visible, escaso residuo fecal líquido; 3 = completamente libre de residuos fecales) (31).

En Colombia son escasos los estudios que evalúen una adecuada preparación intestinal en pacientes de la práctica ordinaria. En este estudio, el objetivo es evaluar la efectividad de dos preparaciones intestinales de bajo volumen, SPMC con respecto al sulfato de sodio/potasio/magnesio, empleados en un hospital de alto nivel, en adultos en un escenario real, con el fin de demostrar la no inferioridad entre los medicamentos.

\section{MATERIALES Y MÉTODOS}

\section{Diseño de estudio}

Se realizó un estudio de cohorte prospectivo, con un grupo de estudio (SPMC) y un grupo comparador (sulfato de sodio/potasio/magnesio), aprobado por el Comité Corporativo de Ética de la Investigación de la Fundación Santa Fe de Bogotá. En este estudio se incluyeron pacientes que aceptaran voluntariamente su participación, después de la explicación amplia de las características del estudio y sus posibles beneficios, manifestado mediante la firma del consentimiento informado.

Este estudio está regido por la Declaración de Helsinki (32) y la Resolución n. 008430 de 1993 de la República de Colombia, Ministerio de Salud (33); como estudio observacional, se considera una investigación que no le agrega riesgo al paciente, teniendo en cuenta que se emplean las preparaciones que se están utilizando en la práctica diaria. Los medicamentos evaluados en este estudio se encuentran registrados en la Superintendencia de Industria y Comercio y el Instituto Nacional de Vigilancia de Medicamentos y Alimentos (INVIMA) (34).

\section{Población de estudio}

Se reclutaron pacientes entre 18-95 años, quienes de forma ambulatoria o intrahospitalaria se les ordenó la realización de colonoscopia para cualquier indicación en un hospital de alto nivel en Bogotá, Colombia, entre mayo de 2019 y diciembre de 2019. Los productos de preparación intestinal utilizados rutinariamente en el hospital son SPMC o sulfato de sodio/potasio/magnesio. Debido a que se propuso un estudio observacional evaluando a los pacientes en el escenario real o vida rutinaria, no se realizó una aleatori- 
zación de la exposición a los medicamentos, por lo cual la decisión del tipo de preparación fue a criterio del médico tratante según las características del paciente para su mayor beneficio clínico, como lo haría en la práctica diaria con el fin de evitar una intervención. Esto se puede comportar como una posible limitación; sin embargo, se realizó un análisis preliminar para mitigar el sesgo de selección.

Los pacientes con colectomía parcial o completa, o aquellos a quienes se les suspendió el procedimiento (por dificultad técnica, dolor o inestabilidad del paciente o alteración anatómica) fueron excluidos del estudio. Los pacientes que realizaron la preparación adicionando algún otro medicamento oral que se hayan preparado durante más de dos días y las mujeres embarazadas o en período de lactancia tampoco fueron incluidos.

\section{Sistematización y análisis de datos}

Todos los pacientes fueron sometidos a un régimen dietético importante para beneficiar la visualización de la mucosa del colon. Adicionalmente, debieron realizar la preparación con alguno de los regímenes de preparación intestinal utilizados en esta institución: 1. SPMC, dosis única; 2. SPMC, dosis dividida; 3. sulfato de sodio/ potasio/magnesio, dosis única; 4. sulfato de sodio/potasio/magnesio, dosis dividida. $\mathrm{Al}$ ingreso del paciente se tomaron datos demográficos y clínicos. Durante el procedimiento se analizó el comportamiento de los diferentes productos de limpieza intestinal por medio de la BBPS. Múltiples estudios consideran una preparación adecuada con una puntuación entre 5 y 7 , según el estudio $(20,29,35)$; en este caso se decidió medir la efectividad del medicamento con un puntaje de referencia $\geq 6$ puntos para una adecuada preparación.

Luego del procedimiento, se interrogó a los pacientes sobre la forma de administración de la preparación, si esta fue completa o incompleta, y de dosis única o dividida. Se identificaron las variables de tolerabilidad del producto empleado por medio de una encuesta de posibles síntomas indeseables y se valoró la presencia de eventos adversos 24 horas después de la administración de las preparaciones. Los pacientes que no completaran el esquema de manera adecuada realizaron una encuesta para identificar los principales factores relacionados con la falta de adherencia a los diferentes tipos de preparación intestinal empleados.

\section{Análisis estadístico}

El cálculo del tamaño de muestra se realizó utilizando OPENEPI (36) estimando el porcentaje de pacientes con preparación adecuada, $86 \%$ y $81 \%$ en cada brazo $(37,38)$, con una desviación estándar de 0,5 , nivel de significancia del $5 \%$ y potencia de la muestra del $80 \%$; calculando un porcentaje de pérdida del $10 \%$. Al ser un estudio de no inferioridad, se considera una diferencia del $3 \%-5 \%$ para lograr un puntaje mínimo de 6 en la escala utilizada. El cálculo dio 520 pacientes para un total de 260 pacientes por cada grupo observacional.

Se ejecutó un análisis exploratorio sobre las variables demográficas y clínicas. Se describieron las variables cualitativas en frecuencias absolutas y relativas, y las variables cuantitativas con promedios, desviación estándar, medianas y rango intercuartílico. Se aplicó la prueba de Shapiro-Wilk para determinar si la distribución era normal o no. Para mitigar el sesgo de selección de muestra debido a la no aleatorización por el tipo de estudio propuesto, se realizó un análisis preliminar y se evaluó la comparabilidad de los grupos observacionales a partir de las características basales de los dos grupos. Se determinó por medio del análisis exploratorio que los dos grupos eran comparables ya que no se evidenciaron diferencias significativas en las características de la población recolectada por el grupo observacional.

Con el análisis bivariado se evaluaron los posibles factores de influencia positiva o negativa en cada preparación intestinal. La distribución de las variables se describió por el desenlace de efectividad. Se realizaron cruces para las variables cualitativas con chi cuadrado $\left(\chi^{2}\right)$ y para las variables cuantitativas con $t$ de Student o con la prueba de U de Mann-Whitney.

Se realizaron logísticas simples para determinar aquellas variables significativas para construir un modelo de análisis multivariado. La efectividad de cada medicamento se evaluó de forma individual mediante un modelo de regresión logística para causalidad. Así mismo, se realizó una serie de regresión logística de modelaje para la evaluación de las variables que se consideró que podían influir sobre la limpieza intestinal de cada medicamento. Posteriormente, se realizó un análisis comparativo de la efectividad del medicamento según la limpieza adecuada por cada segmento del colon (significativo con valores de $p<0,05$ ), para evaluar la hipótesis nula de no inferioridad entre los medicamentos. Para evaluar la tolerabilidad, seguridad y motivos de no cumplimiento se realizó un análisis descriptivo de las variables referentes a estos desenlaces.

\section{RESULTADOS}

\section{Población de estudio}

Se recolectó un total de 598 pacientes que cumplieron los criterios de selección en un período de 7 meses. Al 49 \% (n =293) se les administró SPMC, de los cuales el 90,3\% ( $\mathrm{n}=$ 540) recibió una única dosis el día previo al procedimiento (Tabla 1). 
Tabla 1. Descripción demográfica de la población general $(\mathrm{n}=598)$ comparando entre los dos medicamentos evaluados (SPMC* frente a sulfato de sodio/potasio/magnesio**)

\begin{tabular}{|c|c|c|c|c|c|}
\hline \multicolumn{2}{|c|}{ Variable } & \multicolumn{2}{|c|}{$\begin{array}{c}\text { SPMC } \\
n=293 \\
(49)\end{array}$} & \multicolumn{2}{|c|}{$\begin{array}{c}\text { Sulfato de } \\
\text { sodio, potasio y } \\
\text { magnesio } \\
n=305(51)\end{array}$} \\
\hline & & $\%$ & n & $\%$ & $n$ \\
\hline \multirow[t]{2}{*}{ Sexo } & Mujer & 162 & 55 & 156 & 51 \\
\hline & Hombre & 131 & 45 & 149 & 49 \\
\hline \multirow[t]{3}{*}{ Edad (años) } & $18-49$ & 92 & 31 & 95 & 31 \\
\hline & $50-75$ & 180 & 61 & 183 & 60 \\
\hline & $>75$ & 21 & 7 & 27 & 9 \\
\hline \multirow[t]{3}{*}{ IMC } & $<25$ & 160 & 55 & 174 & 57 \\
\hline & $25-29$ & 101 & 34 & 106 & 35 \\
\hline & $>30$ & 32 & 11 & 25 & 8 \\
\hline \multirow[t]{4}{*}{ Tabaquismo } & Nunca & 205 & 70 & 198 & 65 \\
\hline & Ocasional & 9 & 3 & 7 & 2 \\
\hline & Habitual & 13 & 4 & 15 & 5 \\
\hline & Exfumador & 66 & 23 & 85 & 28 \\
\hline \multirow[t]{4}{*}{ Alcohol } & Nunca & 33 & 11 & 30 & 10 \\
\hline & Ocasional & 239 & 82 & 146 & 48 \\
\hline & Habitual & 19 & 6 & 10 & 3 \\
\hline & Diario & 2 & 1 & 1 & 0 \\
\hline \multirow[t]{5}{*}{ Estado civil } & Soltero/a & 39 & 13 & 36 & 12 \\
\hline & Casado/a & 224 & 76 & 237 & 78 \\
\hline & Separado/a & 17 & 6 & 15 & 5 \\
\hline & Unión libre & 7 & 2 & 3 & 1 \\
\hline & Viudo/a & 6 & 2 & 14 & 5 \\
\hline \multirow{2}{*}{$\begin{array}{l}\text { Antecedente } \\
\text { familiar }\end{array}$} & Sí & 93 & 32 & 105 & 34 \\
\hline & No & 200 & 68 & 200 & 66 \\
\hline \multirow[t]{2}{*}{ Estreñimiento } & Sí & 100 & 34 & 107 & 35 \\
\hline & No & 193 & 66 & 198 & 65 \\
\hline \multirow[t]{2}{*}{ Dieta } & Sí & 289 & 99 & 303 & 99 \\
\hline & No & 4 & 1 & 2 & 1 \\
\hline \multirow{2}{*}{$\begin{array}{l}\text { Preparación } \\
\text { completa }\end{array}$} & Sí & 292 & 100 & 301 & 99 \\
\hline & No & 1 & 0 & 4 & 1 \\
\hline \multirow[t]{2}{*}{ Régimen de dosis } & Dosis única & 244 & 83 & 296 & 97 \\
\hline & Dosis dividida & 49 & 17 & 9 & 3 \\
\hline \multirow[t]{2}{*}{ Comorbilidades } & Sí & 168 & 57 & 184 & 60 \\
\hline & No & 125 & 43 & 121 & 40 \\
\hline
\end{tabular}

\begin{tabular}{|c|c|c|c|c|c|}
\hline \multirow{2}{*}{\multicolumn{2}{|c|}{ Variable }} & \multicolumn{2}{|c|}{$\begin{array}{c}\text { SPMC } \\
n=293 \\
(49)\end{array}$} & \multicolumn{2}{|c|}{$\begin{array}{c}\text { Sulfato de } \\
\text { sodio, potasio y } \\
\text { magnesio } \\
n=305(51)\end{array}$} \\
\hline & & $\%$ & $\mathbf{n}$ & $\%$ & $n$ \\
\hline $\begin{array}{l}\text { Hipertensión } \\
\text { arterial }\end{array}$ & $\begin{array}{l}\text { Sí } \\
\text { No }\end{array}$ & $\begin{array}{c}49 \\
244\end{array}$ & $\begin{array}{l}17 \\
83\end{array}$ & $\begin{array}{c}75 \\
230\end{array}$ & $\begin{array}{l}25 \\
75\end{array}$ \\
\hline Diabetes mellitus & $\begin{array}{l}\text { Sí } \\
\text { No }\end{array}$ & $\begin{array}{c}29 \\
264\end{array}$ & $\begin{array}{l}10 \\
90\end{array}$ & $\begin{array}{c}20 \\
285\end{array}$ & $\begin{array}{c}7 \\
93\end{array}$ \\
\hline Dislipidemia & $\begin{array}{l}\text { Sí } \\
\text { No }\end{array}$ & $\begin{array}{c}49 \\
244\end{array}$ & $\begin{array}{l}17 \\
83\end{array}$ & $\begin{array}{c}38 \\
267\end{array}$ & $\begin{array}{l}12 \\
88\end{array}$ \\
\hline Hipotiroidismo & $\begin{array}{l}\text { Sí } \\
\text { No }\end{array}$ & $\begin{array}{c}62 \\
231\end{array}$ & $\begin{array}{l}21 \\
79\end{array}$ & $\begin{array}{c}61 \\
244\end{array}$ & $\begin{array}{l}20 \\
80\end{array}$ \\
\hline $\begin{array}{l}\text { Tumores no } \\
\text { metastásicos }\end{array}$ & $\begin{array}{l}\text { Sí } \\
\text { No }\end{array}$ & $\begin{array}{c}18 \\
275\end{array}$ & $\begin{array}{c}6 \\
94\end{array}$ & $\begin{array}{c}18 \\
287\end{array}$ & $\begin{array}{c}6 \\
94\end{array}$ \\
\hline $\begin{array}{l}\text { Uso de } \\
\text { antihipertensivos }\end{array}$ & $\begin{array}{l}\text { Sí } \\
\text { No }\end{array}$ & $\begin{array}{c}60 \\
233\end{array}$ & $\begin{array}{l}20 \\
80\end{array}$ & $\begin{array}{c}68 \\
237\end{array}$ & $\begin{array}{l}22 \\
78\end{array}$ \\
\hline $\begin{array}{l}\text { Uso de agentes } \\
\text { hipoglucemiantes }\end{array}$ & $\begin{array}{l}\text { Sí } \\
\text { No }\end{array}$ & $\begin{array}{c}26 \\
267\end{array}$ & $\begin{array}{c}9 \\
91\end{array}$ & $\begin{array}{c}18 \\
287\end{array}$ & $\begin{array}{c}6 \\
94\end{array}$ \\
\hline $\begin{array}{l}\text { Uso de agentes } \\
\text { hipolipemiantes }\end{array}$ & $\begin{array}{l}\text { Sí } \\
\text { No }\end{array}$ & $\begin{array}{c}48 \\
245\end{array}$ & $\begin{array}{l}16 \\
84\end{array}$ & $\begin{array}{c}43 \\
262\end{array}$ & $\begin{array}{l}14 \\
86\end{array}$ \\
\hline Uso de levotiroxina & $\begin{array}{l}\text { Sí } \\
\text { No }\end{array}$ & $\begin{array}{c}61 \\
232\end{array}$ & $\begin{array}{l}21 \\
79\end{array}$ & $\begin{array}{c}60 \\
245\end{array}$ & $\begin{array}{l}20 \\
80\end{array}$ \\
\hline $\begin{array}{l}\text { Uso de } \\
\text { antidepresivos }\end{array}$ & $\begin{array}{l}\text { Sí } \\
\text { No }\end{array}$ & $\begin{array}{c}13 \\
280\end{array}$ & $\begin{array}{c}4 \\
96\end{array}$ & $\begin{array}{c}4 \\
301\end{array}$ & $\begin{array}{c}1 \\
99\end{array}$ \\
\hline Cirugía abdominal & $\begin{array}{l}\text { Sí } \\
\text { No }\end{array}$ & $\begin{array}{l}146 \\
147\end{array}$ & $\begin{array}{l}50 \\
50\end{array}$ & $\begin{array}{l}184 \\
121\end{array}$ & $\begin{array}{l}60 \\
40\end{array}$ \\
\hline Colecistectomía & $\begin{array}{l}\text { Sí } \\
\text { No }\end{array}$ & $\begin{array}{c}31 \\
262\end{array}$ & $\begin{array}{l}11 \\
89\end{array}$ & $\begin{array}{c}50 \\
255\end{array}$ & $\begin{array}{l}16 \\
84\end{array}$ \\
\hline Histerectomía & $\begin{array}{l}\text { Sí } \\
\text { No }\end{array}$ & $\begin{array}{c}30 \\
263\end{array}$ & $\begin{array}{l}10 \\
90\end{array}$ & $\begin{array}{c}38 \\
267\end{array}$ & $\begin{array}{l}12 \\
88\end{array}$ \\
\hline Cesárea & $\begin{array}{l}\text { Sí } \\
\text { No }\end{array}$ & $\begin{array}{c}45 \\
248\end{array}$ & $\begin{array}{l}15 \\
85\end{array}$ & $\begin{array}{l}37 \\
268\end{array}$ & $\begin{array}{l}12 \\
88\end{array}$ \\
\hline Cirugía plástica & $\begin{array}{l}\text { Sí } \\
\text { No }\end{array}$ & $\begin{array}{c}8 \\
285\end{array}$ & $\begin{array}{c}3 \\
97\end{array}$ & $\begin{array}{c}24 \\
281\end{array}$ & $\begin{array}{c}8 \\
92\end{array}$ \\
\hline
\end{tabular}

*Travad PIK. **Izinova.

\section{Desenlace de la limpieza intestinal completa}

El SPMC obtuvo un promedio de 6,98 $\pm 1,86$ con el $78 \%$ (n $=228$ ) de limpieza adecuada (BBPS $\geq 6$ puntos); mientras que el sulfato de sodio/potasio/magnesio obtuvo un prome-

dio de $7,39 \pm 1,83$, de los cuales el $83 \%(n=254)$ obtuvo una adecuada limpieza intestinal, con diferencia no significativa $(p$ $=0,649)$ y dentro del margen definido como no inferioridad.

Se demostró que para SPMC, los hombres obtuvieron un promedio total de Boston y limpieza adecuada significati- 
vamente menor al de las mujeres $(6,69 \pm 1,91 ; 95$ [42\%]; Odds ratio $[\mathrm{OR}]: 0,47[0,24-0,94] ; p=0,034)$. Así mismo, se demostró que pacientes con mayor índice de masa corporal (IMC; > 30) obtuvieron un peor rendimiento en la limpieza intestinal $(6,44 \pm 2,14$; OR 0,63 [0,41-0,98]; $p=0,039)$. Los pacientes con comorbilidades en general no presentaron diferencias significativas; sin embargo, los pacientes específicamente con diabetes mellitus demostraron un peor desempeño en la limpieza intestinal $(6,14 \pm 2,42 ; 16$ [7\%]; OR: $0,29[0,12-0,76] ; p=0,012)$ (Tabla 2).

Para el sulfato de sodio/potasio/magnesio, los pacientes de estado civil soltero alcanzaron un promedio total

Tabla 2. Regresión logística multivariable que evalúa la escala de Boston promedio y variables con adecuada preparación intestinal para SPMC $(\mathrm{n}=293$ )

\begin{tabular}{|c|c|c|c|c|c|}
\hline \multicolumn{2}{|c|}{ Variable } & Promedio $\pm \mathrm{DE}$ & $\begin{array}{l}\text { Limpieza intestinal adecuada } \\
\mathrm{n}(\%)\end{array}$ & Valor $p$ & OR (IC $95 \%)$ \\
\hline Medicamento & & $6,98 \pm 1,86$ & $228(78)$ & 0,649 & $1,63(0,2-13,5)$ \\
\hline Sexo & $\begin{array}{l}\text { Mujer } \\
\text { Hombre }\end{array}$ & $\begin{array}{l}7,2 \pm 1,79 \\
6,69 \pm 1,91\end{array}$ & $\begin{array}{l}133(58) \\
95(42)\end{array}$ & $\begin{array}{c}\text { Referencia } \\
0,034\end{array}$ & $\begin{array}{c}\text { Referencia } \\
0,47(0,24-0,94)\end{array}$ \\
\hline Edad (años) & $\begin{array}{l}18-49 \\
50-75 \\
>75\end{array}$ & $\begin{array}{l}7,22 \pm 1,66 \\
6,93 \pm 1,91 \\
6,38 \pm 2,09\end{array}$ & $\begin{array}{c}75(33) \\
139(61) \\
14(6)\end{array}$ & $\begin{array}{c}\text { Referencia } \\
0,965 \\
0,947\end{array}$ & $\begin{array}{c}\text { Referencia } \\
0,99(0,51-1,89) \\
0,98(0,51-1,87)\end{array}$ \\
\hline IMC & $\begin{array}{l}<25 \\
25-29 \\
>30\end{array}$ & $\begin{array}{c}7,15 \pm 1,83 \\
6,88 \pm 1,8 \\
6,44 \pm 2,14\end{array}$ & $\begin{array}{l}128(56) \\
77(34) \\
23(10)\end{array}$ & $\begin{array}{c}\text { Referencia } \\
0,170 \\
0,039\end{array}$ & $\begin{array}{c}\text { Referencia } \\
0,74(0,47-1,14) \\
0,63(0,41-0,98)\end{array}$ \\
\hline Alcohol & $\begin{array}{l}\text { Nunca } \\
\text { Ocasional } \\
\text { Habitual } \\
\text { Diario }\end{array}$ & $\begin{array}{c}7,03 \pm 1,9 \\
7,02 \pm 1,81 \\
6,58 \pm 2,19 \\
5,5 \pm 4,95\end{array}$ & $\begin{array}{c}27(12) \\
186(82) \\
14(6) \\
1(0)\end{array}$ & $\begin{array}{c}\text { Referencia } \\
0,514 \\
0,192 \\
0,050\end{array}$ & $\begin{array}{c}\text { Referencia } \\
0,8(0,41-1,55) \\
0,64(0,33-1,25) \\
0,52(0,27-1,00)\end{array}$ \\
\hline Estado civil & $\begin{array}{l}\text { Soltero/a } \\
\text { Casado/a } \\
\text { Separado/a } \\
\text { Unión libre } \\
\text { Viudo/a }\end{array}$ & $\begin{array}{c}7,23 \pm 1,86 \\
6,92 \pm 1,89 \\
6,76 \pm 1,89 \\
7,86 \pm 1,21 \\
7 \pm 1,26\end{array}$ & $\begin{array}{c}31(14) \\
170(75) \\
14(6) \\
7(3) \\
6(3)\end{array}$ & $\begin{array}{c}\text { Referencia } \\
\quad 0,003 \\
<0,001 \\
<0,001 \\
<0,001\end{array}$ & $\begin{array}{c}\text { Referencia } \\
2,51(1,38-4,58) \\
3,99(2,19-7,25) \\
6,32(3,47-11,5) \\
10,02(5,51-18,24)\end{array}$ \\
\hline Antecedente familiar & $\begin{array}{l}\text { No } \\
\text { Sí }\end{array}$ & $\begin{array}{l}6,95 \pm 1,78 \\
7,04 \pm 2,04\end{array}$ & $\begin{array}{l}158(69) \\
70(31)\end{array}$ & $\begin{array}{c}\text { Referencia } \\
0,524\end{array}$ & $\begin{array}{c}\text { Referencia } \\
0,82(0,436-1,53)\end{array}$ \\
\hline Régimen de dosis & $\begin{array}{l}\text { Dosis única } \\
\text { Dosis dividida }\end{array}$ & $\begin{array}{c}6,9 \pm 1,89 \\
7,39 \pm 1,63\end{array}$ & $\begin{array}{c}211(93) \\
17(7)\end{array}$ & $\begin{array}{l}0,629 \\
0,334\end{array}$ & $\begin{array}{l}0,76(0,26-2,29) \\
0,58(0,19-1,74)\end{array}$ \\
\hline Comorbilidades & $\begin{array}{l}\text { No } \\
\text { Sí }\end{array}$ & $\begin{array}{l}7,18 \pm 1,72 \\
6,83 \pm 1,95\end{array}$ & $\begin{array}{l}104(46) \\
124(54)\end{array}$ & $\begin{array}{c}\text { Referencia } \\
0,391\end{array}$ & $\begin{array}{c}\text { Referencia } \\
0,72(0,34-1,53)\end{array}$ \\
\hline Hipertensión arterial & $\begin{array}{l}\text { No } \\
\text { Sí }\end{array}$ & $\begin{array}{c}6,9 \pm 1,89 \\
7,39 \pm 1,63\end{array}$ & $\begin{array}{l}179(79) \\
49(21)\end{array}$ & $\begin{array}{c}\text { Referencia } \\
0,647\end{array}$ & $\begin{array}{c}\text { Referencia } \\
1,22(0,52-2,84)\end{array}$ \\
\hline Diabetes mellitus & $\begin{array}{l}\text { No } \\
\text { Sí }\end{array}$ & $\begin{array}{l}7,07 \pm 1,77 \\
6,14 \pm 2,42\end{array}$ & $\begin{array}{c}212(93) \\
16(7)\end{array}$ & $\begin{array}{c}\text { Referencia } \\
0,012\end{array}$ & $\begin{array}{c}\text { Referencia } \\
0,29(0,12-0,76)\end{array}$ \\
\hline Uso de antidepresivos & $\begin{array}{l}\text { No } \\
\text { Sí }\end{array}$ & $\begin{array}{l}6,99 \pm 1,85 \\
6,69 \pm 2,02\end{array}$ & $\begin{array}{c}220(96) \\
8(4)\end{array}$ & $\begin{array}{c}\text { Referencia } \\
0,300\end{array}$ & $\begin{array}{c}\text { Referencia } \\
0,51(0,14-1,84)\end{array}$ \\
\hline Cirugía abdominal & $\begin{array}{l}\text { No } \\
\text { Sí }\end{array}$ & $\begin{array}{l}6,94 \pm 1,79 \\
7,02 \pm 1,94\end{array}$ & $\begin{array}{l}118(52) \\
110(48)\end{array}$ & $\begin{array}{c}\text { Referencia } \\
0,636\end{array}$ & $\begin{array}{c}\text { Referencia } \\
0,34(0,4-1,74)\end{array}$ \\
\hline Colecistectomía & $\begin{array}{l}\text { No } \\
\text { Sí }\end{array}$ & $\begin{array}{l}7,08 \pm 1,75 \\
6,16 \pm 2,48\end{array}$ & $\begin{array}{c}210(92) \\
18(8)\end{array}$ & $\begin{array}{c}\text { Referencia } \\
0,083\end{array}$ & $\begin{array}{c}\text { Referencia } \\
0,43(0,17-1,12)\end{array}$ \\
\hline Histerectomía & $\begin{array}{l}\text { No } \\
\text { Sí }\end{array}$ & $\begin{array}{l}7,01 \pm 1,85 \\
6,73 \pm 1,93\end{array}$ & $\begin{array}{c}207(91) \\
21(9)\end{array}$ & $\begin{array}{c}\text { Referencia } \\
0,056\end{array}$ & $\begin{array}{c}\text { Referencia } \\
0,36(0,12-1,03)\end{array}$ \\
\hline
\end{tabular}

DE: desviación estándar; IC: intervalo de confianza. 
de Boston y limpieza adecuada $(7,23 \pm 1,86,31$ [14 \%]; OR: $1,87[1,04-3,36] ; p=0,038)$. Los pacientes con cirugías abdominales no presentaron diferencias significativas; sin embargo, los pacientes con colecistectomía demostraron un peor desempeño en la limpieza intestinal $(6,16 \pm 2,48 ; 18[8 \%]$; OR: $0,38[0,16-0,92] ; p=$ $0,032)$. Ninguna otra variable demostró una relación significativa (Tabla 3).

Tabla 3. Regresión logística multivariable que evalúa la escala de Boston promedio y variables con una adecuada preparación intestinal para sulfato de sodio, potasio y magnesio $(\mathrm{n}=305)$

\begin{tabular}{|c|c|c|c|c|c|}
\hline \multicolumn{2}{|c|}{ Variable } & Promedio $\pm D E$ & Limpieza intestinal adecuada & Valor $p$ & OR (IC $95 \%)$ \\
\hline \multicolumn{2}{|l|}{ Medicamento } & $7,39 \pm 1,83$ & $254(83)$ & Referencia & Referencia \\
\hline Sexo & $\begin{array}{l}\text { Mujer } \\
\text { Hombre }\end{array}$ & $\begin{array}{c}7,2 \pm 1,79 \\
6,69 \pm 1,91\end{array}$ & $\begin{array}{c}133(58) \\
95(42)\end{array}$ & $\begin{array}{c}\text { Referencia } \\
0,765\end{array}$ & $\begin{array}{c}\text { Referencia } \\
1,13(0,52-2,47)\end{array}$ \\
\hline Edad (años) & $\begin{array}{l}18-49 \\
50-75 \\
>75\end{array}$ & $\begin{array}{l}7,22 \pm 1,66 \\
6,93 \pm 1,91 \\
6,38 \pm 2,09\end{array}$ & $\begin{array}{c}75(33) \\
139(61) \\
14(6)\end{array}$ & $\begin{array}{c}\text { Referencia } \\
0,536 \\
0,354\end{array}$ & $\begin{array}{c}\text { Referencia } \\
1,22(0,65-2,30) \\
1,35(0,72-2,54)\end{array}$ \\
\hline IMC & $\begin{array}{l}<25 \\
25-29 \\
>30\end{array}$ & $\begin{array}{c}7,15 \pm 1,83 \\
6,88 \pm 1,8 \\
6,44 \pm 2,14\end{array}$ & $\begin{array}{l}128(56) \\
77(34) \\
23(10)\end{array}$ & $\begin{array}{c}\text { Referencia } \\
0,252 \\
0,086\end{array}$ & $\begin{array}{c}\text { Referencia } \\
0,75(0,45-1,23) \\
0,64(0,39-1,06)\end{array}$ \\
\hline Alcohol & $\begin{array}{l}\text { Nunca } \\
\text { Ocasional } \\
\text { Habitual } \\
\text { Diario }\end{array}$ & $\begin{array}{c}7,03 \pm 1,9 \\
7,02 \pm 1,81 \\
6,58 \pm 2,19 \\
5,5 \pm 4,95\end{array}$ & $\begin{array}{c}27(12) \\
186(82) \\
14(6) \\
1(0)\end{array}$ & $\begin{array}{c}\text { Referencia } \\
0,518 \\
0,197 \\
0,053\end{array}$ & $\begin{array}{c}\text { Referencia } \\
0,76(0,33-1,76) \\
0,57(0,25-1,33) \\
0,43(0,19-1,01)\end{array}$ \\
\hline Estado civil & $\begin{array}{l}\text { Soltero/a } \\
\text { Casado/a } \\
\text { Separado/a } \\
\text { Unión libre } \\
\text { Viudo/a }\end{array}$ & $\begin{array}{c}7,23 \pm 1,86 \\
6,92 \pm 1,89 \\
6,76 \pm 1,89 \\
7,86 \pm 1,21 \\
7 \pm 1,26\end{array}$ & $\begin{array}{c}31(14) \\
170(75) \\
14(6) \\
7(3) \\
6(3)\end{array}$ & $\begin{array}{c}\text { Referencia } \\
<0,001 \\
<0,001 \\
<0,001 \\
<0,001\end{array}$ & $\begin{array}{c}\text { Referencia } \\
3,48(1,93-6,28) \\
6,5(3,61-11,7) \\
12,1(6,74-21,9) \\
22,7(12,6-40,8)\end{array}$ \\
\hline Antecedente familiar & $\begin{array}{l}\text { No } \\
\text { Sí }\end{array}$ & $\begin{array}{l}6,95 \pm 1,78 \\
7,04 \pm 2,04\end{array}$ & $\begin{array}{l}158(69) \\
70(31)\end{array}$ & $\begin{array}{c}\text { Referencia } \\
0,115\end{array}$ & $\begin{array}{c}\text { Referencia } \\
1,81(0,89-3,79)\end{array}$ \\
\hline Régimen de dosis & $\begin{array}{l}\text { Dosis única } \\
\text { Dosis dividida }\end{array}$ & $\begin{array}{c}6,9 \pm 1,89 \\
7,39 \pm 1,63\end{array}$ & $\begin{array}{c}211(93) \\
17(7)\end{array}$ & $\begin{array}{l}0,574 \\
0,260\end{array}$ & $\begin{array}{c}0,62(0,12-3,3) \\
0,38(0,07-2,04)\end{array}$ \\
\hline Comorbilidades & $\begin{array}{l}\text { No } \\
\text { Sí }\end{array}$ & $\begin{array}{l}7,18 \pm 1,72 \\
6,83 \pm 1,95\end{array}$ & $\begin{array}{l}104(46) \\
124(54)\end{array}$ & $\begin{array}{c}\text { Referencia } \\
0,611\end{array}$ & $\begin{array}{c}\text { Referencia } \\
0,8(0,37-1,79)\end{array}$ \\
\hline Dislipidemia & $\begin{array}{l}\text { No } \\
\text { Sí }\end{array}$ & $\begin{array}{c}7,43 \pm 1,77 \\
7,1 \pm 2,21\end{array}$ & $\begin{array}{c}225(89) \\
29(11)\end{array}$ & $\begin{array}{c}\text { Referencia } \\
0,418\end{array}$ & $\begin{array}{c}\text { Referencia } \\
0,68(0,27-1,73)\end{array}$ \\
\hline Hipertensión arterial & $\begin{array}{l}\text { No } \\
\text { Sí }\end{array}$ & $\begin{array}{r}6,9 \pm 1,89 \\
7,39 \pm 1,63\end{array}$ & $\begin{array}{l}179(79) \\
49(21)\end{array}$ & $\begin{array}{c}\text { Referencia } \\
0,839\end{array}$ & $\begin{array}{c}\text { Referencia } \\
0,92(0,39-2,16)\end{array}$ \\
\hline Diabetes mellitus & $\begin{array}{l}\text { No } \\
\text { Sí }\end{array}$ & $\begin{array}{l}7,07 \pm 1,77 \\
6,14 \pm 2,42\end{array}$ & $\begin{array}{c}212(93) \\
16(7)\end{array}$ & $\begin{array}{c}\text { Referencia } \\
0,895\end{array}$ & $\begin{array}{c}\text { Referencia } \\
1,09(0,3-3,99)\end{array}$ \\
\hline Cirugía abdominal & $\begin{array}{l}\text { No } \\
\text { Sí }\end{array}$ & $\begin{array}{l}6,94 \pm 1,79 \\
7,02 \pm 1,94\end{array}$ & $\begin{array}{l}118(52) \\
110(48)\end{array}$ & $\begin{array}{c}\text { Referencia } \\
0,342\end{array}$ & $\begin{array}{c}\text { Referencia } \\
1,5(0,65-3,46)\end{array}$ \\
\hline Colecistectomía & $\begin{array}{l}\text { No } \\
\text { Sí }\end{array}$ & $\begin{array}{l}7,08 \pm 1,75 \\
6,16 \pm 2,48\end{array}$ & $\begin{array}{c}210(92) \\
18(8)\end{array}$ & $\begin{array}{c}\text { Referencia } \\
0,032\end{array}$ & $\begin{array}{c}\text { Referencia } \\
0,38(0,16-0,92)\end{array}$ \\
\hline Histerectomía & $\begin{array}{l}\text { No } \\
\text { Sí }\end{array}$ & $\begin{array}{l}7,01 \pm 1,85 \\
6,73 \pm 1,93\end{array}$ & $\begin{array}{c}207(91) \\
21(9)\end{array}$ & $\begin{array}{c}\text { Referencia } \\
0,853\end{array}$ & $\begin{array}{c}\text { Referencia } \\
0,89(0,28-2,84)\end{array}$ \\
\hline Cesárea & $\begin{array}{l}\text { No } \\
\text { Sí }\end{array}$ & $\begin{array}{l}7,39 \pm 1,81 \\
7,35 \pm 2,02\end{array}$ & $\begin{array}{c}224(88) \\
30(12)\end{array}$ & $\begin{array}{c}\text { Referencia } \\
0,443\end{array}$ & $\begin{array}{c}\text { Referencia } \\
0,65(0,22-1,94)\end{array}$ \\
\hline Cirugía plástica & $\begin{array}{l}\text { No } \\
\text { Sí }\end{array}$ & $\begin{array}{l}7,39 \pm 1,84 \\
7,38 \pm 1,74\end{array}$ & $\begin{array}{c}234(92) \\
20(8)\end{array}$ & $\begin{array}{c}\text { Referencia } \\
0,767\end{array}$ & $\begin{array}{c}\text { Referencia } \\
0,82(0,22-3,06)\end{array}$ \\
\hline
\end{tabular}




\section{Desenlace de la limpieza intestinal por segmento del colon}

Para el colon izquierdo, el $48 \%(n=267)$ de pacientes con SPMC tuvo una adecuada limpieza con respecto al sulfato de sodio/potasio/magnesio $(\mathrm{n}=288$ [52]; OR: $1,65[0,88-3,11] ; p=0,154)$; para el colon trasverso se evidenció un mejor resultado con SPMC $(\mathrm{n}=273[54 \%]$; OR: $2,11[1,32-3,35] ; p=0,002)$; y para colon derecho los resultados fueron similares $(\mathrm{n}=238$ [52\%]; OR: 1,12 $[0,74-1,71] ; p=0,583$ ) (Tabla 4). Adicionalmente, se realizó una comparación de la cantidad de pacientes que obtuvo un puntaje de 0-1 en algún segmento del colon, pese a tener un puntaje global de BBPS $\geq 6$ puntos. Se evidenció que, de los pacientes preparados con SPMC, el 0,44\% tuvo un puntaje de $0-1$ con un global $\geq 6$, respecto al $0,39 \%$ de sulfato de sodio/potasio/magnesio $(p=0,469)$; en colon trasverso, los resultados fueron $3,07 \%$ frente a $1,97 \%$ $(p=0,222)$, respectivamente; $\mathrm{y}$ en colon derecho fueron $7,02 \%$ frente a $4,33 \%(p=0,1025)$, respectivamente.

\section{Tolerabilidad de la preparación intestinal}

De los 598 pacientes, el $1 \%(\mathrm{n}=6)$ no completó la preparación. Los pacientes expuestos a SPMC completaron la preparación en un 99,3\% $(\mathrm{n}=291)$; de los pacientes expuestos a sulfato de sodio/potasio/magnesio, el 98,7 \% $(\mathrm{n}=301)$ completó la preparación. $\mathrm{Al}$ evaluar la presencia y frecuencia de los síntomas no deseados y eventos adversos, se demostró una diferencia significativa $(\mathrm{p}<0,001)$, en la que el SPMC tuvo una mejor tolerancia (Figura 1). Se realizó un análisis de correspondencia en el que se evidencian los síntomas más frecuentes según el grupo observacional. Los pacientes a los que se les administró SPMC evidenciaron con más frecuencia cefalea, boca seca y taquicardia; en cambio, los que recibieron sulfato de sodio/potasio/magnesio culminaron con una mayor tasa de alteraciones neurológicas y somnolencia.
El dolor y la distensión abdominal fueron síntomas comunes en ambos grupos observacionales (Figura 2). Dentro de los motivos por los cuales se suspendió el procedimiento se encuentra la intolerancia del paciente debido a dolor u otras alteraciones anatómicas (Figura 3).

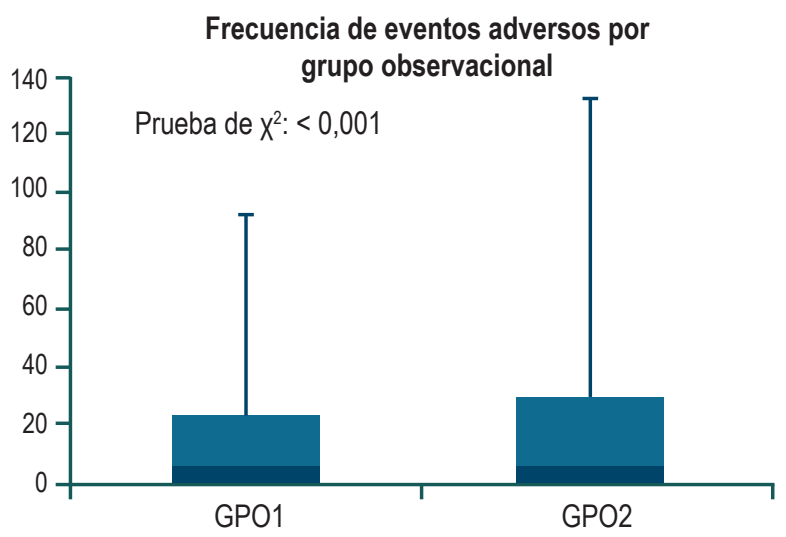

Figura 1. Frecuencia de los eventos adversos (sintomatología no deseable) que se identificaron por cada grupo observacional. GPO1: grupo observacional 1 (SPMC, Travad PIK). GPO2: grupo observacional 2 (sulfato de sodio, potasio y magnesio, Izinova).

Se evaluó el promedio de edades según la preparación intestinal empleada y la aparición de eventos adversos y se observó que, de los pacientes que presentaron eventos adversos con SPMC, el promedio de edad fue 54,6 años, comparado con 53,3 años en promedio de los pacientes con sulfato de sodio/potasio/magnesio. En la evaluación de cada tipo de evento adverso acorde al medicamento, todos los promedios de edades estaban por debajo de 65 años, excepto por un paciente de 68 años con SPMC que presentó una alteración neurológica. Además, se valoró la proporción de pacientes $\geq 65$ años con efectos adversos de SPMC $(\mathrm{n}=25[27 \%])$ y de sulfato de sodio/potasio/magnesio $(\mathrm{n}=33[25 \%])(p=0,309)$.

Tabla 4. Regresión logística multivariable que evalúa la adecuada preparación intestinal y las preparaciones intestinales según cada segmento del colon

\begin{tabular}{|c|c|c|c|c|c|c|c|c|c|}
\hline \multirow{2}{*}{$\begin{array}{l}\text { Preparación } \\
\text { intestinal }\end{array}$} & \multicolumn{3}{|c|}{ Colon izquierdo } & \multicolumn{3}{|c|}{ Colon transverso } & \multicolumn{2}{|c|}{ Colon derecho } & \multirow[t]{2}{*}{ Valor $p$} \\
\hline & $\begin{array}{l}\text { Limpieza } \\
\text { intestinal } \\
\text { adecuada } \\
\text { n (\%) }\end{array}$ & OR (IC $95 \%$ ) & Valor $p$ & $\begin{array}{l}\text { Limpieza } \\
\text { intestinal } \\
\text { adecuada } \\
\mathbf{n}(\%)\end{array}$ & OR (IC $95 \%)$ & Valor $p$ & $\begin{array}{c}\text { Limpieza } \\
\text { intestinal } \\
\text { adecuada } \\
\text { n (\%) }\end{array}$ & OR (IC $95 \%$ ) & \\
\hline SPMC & $267(48)$ & Referencia & Referencia & $235(46)$ & Referencia & Referencia & $253(48)$ & Referencia & Referencia \\
\hline $\begin{array}{l}\text { Sulfato de } \\
\text { sodio, potasio y } \\
\text { magnesio }\end{array}$ & $288(52)$ & $1,65(0,88-3,11)$ & 0,154 & $273(54)$ & $2,11(1,32-3,35)$ & 0,002 & $238(52)$ & $1,12(0,74-1,71)$ & 0,583 \\
\hline
\end{tabular}


Eventos adversos por grupo observacional, análisis de correspondencia combinado

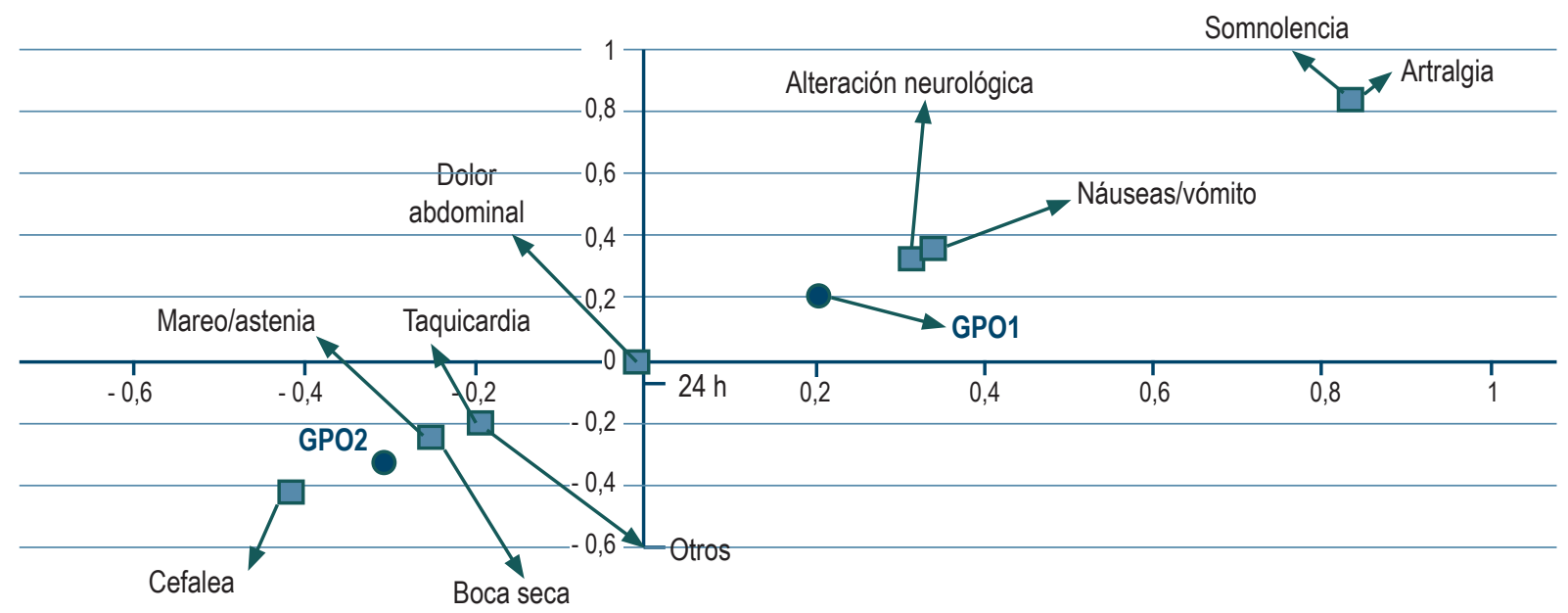

Figura 2. Análisis de correspondencia combinado de los eventos adversos (sintomatología no deseable) por grupo observacional. GPO1: grupo observacional 1 (SPMC, Travad PIK). GPO2: grupo observacional 2 (sulfato de sodio, potasio y magnesio, Izinova).

\section{Motivos de suspensión del procedimiento por grupo observacional}

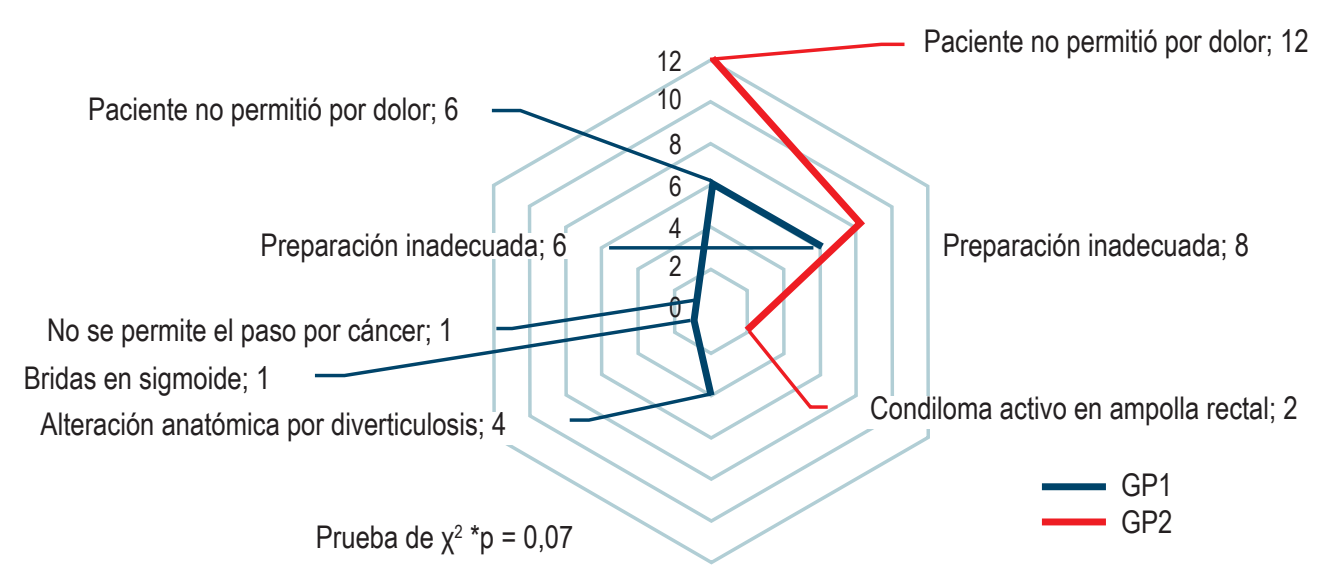

Figura 3. Razones por las cuales los pacientes no lograron el procedimiento. *Asociación significativa al $10 \%$. GPO1: grupo observacional 1 (SPMC, Travad PIK). GPO2: grupo observacional 2 (sulfato de sodio, potasio y magnesio, Izinova).

\section{DISCUSIÓN}

La preparación intestinal es de vital importancia para la realización adecuada de la colonoscopia que permitirá la identificación de patologías colónicas de forma oportuna. La evaluación de los diferentes productos empleados para este fin ha sido prioridad para muchos expertos en el tema. Schreiber y colaboradores (13) evaluaron la eficacia y seguridad del NER1006 (PEG) y demostraron la no inferioridad frente a SPMC. Los pacientes expuestos a NER1006 experimentaron más eventos adversos y mostraron una menor adherencia al tratamiento. A pesar de estar compa- rando agentes de alto volumen con uno de bajo volumen, demostraron resultados similares a este estudio.

$\mathrm{Gu}$ y colaboradores (37) realizaron un estudio observacional comparativo de efectividad y tolerabilidad de las preparaciones disponibles en pacientes en la vida real en Los Ángeles, California. Todos los resultados fueron comparados con Golytely (PEG 3350), preparación estándar según la Asociación Americana de Endoscopia Gastrointestinal (ASGE). El puntaje global de Boston fue significativamente mayor $(\geq 7)$ para Miralax $(p=0,001)$, Suprep $(p=0,001)$ y Moviprep $(p=0,004)$; estos medicamentos tienen como componente activo SPMC y demostraron puntajes de 
Boston similares a los de este estudio. Prepopik $(99,1 \%)$ y el citrato de magnesio $(98,1 \%)$ fueron mejor tolerados que Golytely (82,9\%), aunque sin diferencias significativas en la limpieza intestinal. Este se realizó en un escenario real, arrojando resultados similares a los de este estudio. La evidencia en la vida real busca validar los ensayos clínicos (39) con estudios que permitan evaluar el comportamiento de determinado medicamento en la atención de rutina.

Un estudio reciente comparó la satisfacción del paciente ante dos agentes de bajo volumen: solución de sulfato oral (OSS) y SPMC. Los dos grupos indicaron que estaban dispuestos a someterse a una colonoscopia repetida con el mismo laxante en el $91 \%$ y $93 \%$ de los casos, respectivamente. Sin embargo, el grupo de SPMC superó significativamente al grupo OSS $(p=0,006)$. Las quejas más comunes fueron distensión y dolor abdominal ( $16,7 \%$ frente a $10,2 \%$ para los grupos OSS frente a SPMC) (40). En este estudio también existió una diferencia significativa $(p<0,001)$ en cuanto a la tolerabilidad de los pacientes frente a las diferentes preparaciones. Se demostraron resultados similares en el tipo y frecuencia de síntomas en los medicamentos; las náuseas y emesis no son síntomas comunes para el SPMC.

En Colombia no se han realizado estudios de la efectividad del SPMC poscomercializado. Dicho estudio puede fortalecer y enriquecer la literatura médica para la decisión de los diferentes tipos de preparación intestinal. Los costos en nuestro estudio no se evaluaron; no obstante, realizar un estudio de costo-efectividad a futuro generaría un mayor impacto con respecto a qué medicamento es mejor usar en determinados casos según su costo. Esto podría ser una evaluación interesante en ciertas regiones de América Latina, donde se encuentran países en vía de desarrollo y, particularmente, países como Colombia, cuyo sistema de salud existente tiende a dificultar el acceso a algunos medicamentos; esto entorpece la prevención de determinadas enfermedades, lo que obliga al médico a actuar frente a enfermedades ya establecidas e incluso muchas veces en estado avanzado.
$\mathrm{Al}$ ser un estudio de único centro, pueden surgir posibles diferencias entre los hallazgos encontrados en este estudio con respecto a otros estudios. Sin embargo, se cree que el tamaño de la muestra fue importante y significativa para el objetivo del estudio; se considera que los estudios con un mayor tamaño de muestra no tienen variaciones significativas en sus resultados.

Este estudio tiene un impacto importante a nivel de salud pública y brinda un beneficio global en cuanto al CCR, ya que, a pesar de no identificar una diferencia significativa en la efectividad de las preparaciones evaluadas, por medio de este estudio sí se encontró una mayor adherencia al SPMC. Esto permite que los pacientes realicen la preparación completa con mayor agrado, lo que aumenta las probabilidades de éxito en la limpieza intestinal y determina el mejor método de limpieza intestinal para aumentar la tasa de detección de pólipos tempranamente y realizar un manejo oportuno. Los estudios de costo-efectividad podrían ayudar a definir el mejor medicamento según las necesidades de la población.

\section{Agradecimientos}

No aplica.

\section{Fuente de financiación}

Los autores no participaron en ninguna convocatoria ni recibieron ningún soporte financiero de su institución profesional o educativa. Tampoco recibieron fondos o recursos de ninguna otra entidad durante el desarrollo del estudio ni del manuscrito.

\section{Conflictos de interés}

Los autores declaran no tener ningún conflicto de interés que pueda afectar los resultados de este estudio.

\section{REFERENCIAS}

1. Voiosu T, Tanțău A, Voiosu A, Benguş A, Mocanu C, Smarandache B, Baicuş C, Vişovan I, Mateescu B. Preparation regimen is more important than patient-related factors: a randomized trial comparing a standard bowel preparation before colonoscopy with an individualized approach. Rom J Intern Med. 2017;55(1):36-43. https://doi.org/10.1515/rjim-2016-0047

2. Bray F, Ferlay J, Soerjomataram I, Siegel RL, Torre LA, Jemal A. Global cancer statistics 2018: GLOBOCAN esti- mates of incidence and mortality worldwide for 36 cancers in 185 countries. CA Cancer J Clin. 2018;68(6):394-424. https://doi.org/10.3322/caac.21492

3. Galán E, Puerto D, Salazar L, Oliveros R, Arredondo L. Manual para la detección temprana del cáncer de colon y recto. Bogotá: Instituto Nacional de Cancerología; 2015.

4. Maratt JK, Calderwood AH. Colorectal Cancer Screening and Surveillance Colonoscopy in Older Adults. Curr Treat 
Options Gastroenterol. 2019;17(2):292-302.

https://doi.org/10.1007/s1 1938-019-00230-9

5. Atkin WS, Edwards R, Kralj-Hans I, Wooldrage K, Hart AR, Northover JM, Parkin DM, Wardle J, Duffy SW, Cuzick J; UK Flexible Sigmoidoscopy Trial Investigators. Once-only flexible sigmoidoscopy screening in prevention of colorectal cancer: a multicentre randomised controlled trial. The Lancet. 2010;375(9726):1624-33. https://doi.org/10.1016/S0140-6736(10)60551-X

6. Baxter NN, Warren JL, Barrett MJ, Stukel TA, Doria-Rose VP. Association Between Colonoscopy and Colorectal Cancer Mortality in a US Cohort According to Site of Cancer and Colonoscopist Specialty. J Clin Oncol. 2012;30(21):2664-9. https://doi.org/10.1200/JCO.2011.40.4772

7. Segnan N, Armaroli P, Bonelli L, Risio M, Sciallero S, Zappa M, Andreoni B, Arrigoni A, Bisanti L, Casella C, Crosta C, Falcini F, Ferrero F, Giacomin A, Giuliani O, Santarelli A, Visioli CB, Zanetti R, Atkin WS, Senore C; SCORE Working Group. Once-Only Sigmoidoscopy in Colorectal Cancer Screening: Follow-up Findings of the Italian Randomized Controlled Trial--SCORE. JNCI J Natl Cancer Inst. 2011;103(17):1310-22.

https://doi.org/10.1093/jnci/djr284

8. Flemming JA, Vanner SJ, Hookey LC. Split-dose picosulfate, magnesium oxide, and citric acid solution markedly enhances colon cleansing before colonoscopy: a randomized, controlled trial. Gastrointest Endosc. 2012;75(3):537544.e1.

https://doi.org/10.1016/j.gie.2011.09.018

9. le Clercq CMC, Bouwens MWE, Rondagh EJA, Bakker CM, Keulen ETP, de Ridder RJ, Winkens B, Masclee AA, Sanduleanu S. Postcolonoscopy colorectal cancers are preventable: a population-based study. Gut. 2014;63(6):957-63. https://doi.org/10.1136/gutjnl-2013-304880

10. Cortes BGW, Cabral RM, Carmo GAA do, Queiroz FL de, Leite SM de O, Andrade AC de S, Ferreira A, Alcici MA, Cortes M. Double blinded randomized clinical trial to assess the effectiveness of several preparations for colonoscopy. J Coloproctology. 2018;38(4):302-8. https://doi.org/10.1016/j.jcol.2018.07.001

11. Hassan C, Rossi PG, Camilloni L, Rex DK, JimenezCendales B, Ferroni E, Borgia P, Zullo A, Guasticchi G; HTA Group. Meta-analysis: adherence to colorectal cancer screening and the detection rate for advanced neoplasia, according to the type of screening test. Aliment Pharmacol Ther. 2012;36(10):929-40. https://doi.org/10.1111/apt.12071

12. Marzo M Castells, A, Bellas B. Guía de práctica clínica sobre la prevención del cáncer colorrectal. Gastroentorol Hepatol. 2004; 10:579-634. https://doi.org/10.1016/S0210-5705(03)70535-4

13. Schreiber S, Baumgart D, Drenth J, Filip R, Clayton L, Hylands K, Berton A, Salvatore G, Denaro V. Colon cleansing efficacy and safety with 1 L NER1006 versus sodium picosulfate with magnesium citrate: a randomized phase 3 trial. Endoscopy. 2019;51(01):73-84.

https://doi.org/10.1055/a-0639-5070

14. Oh CH, Lee CK, Kim J-W, Shim J-J, Jang JY. Suboptimal Bowel Preparation Significantly Impairs Colonoscopic Detection of Non-polypoid Colorectal Neoplasms. Dig Dis Sci. 2015;60(8):2294-303. https://doi.org/10.1007/s10620-015-3628-6

15. Lieberman DA, Rex DK, Winawer SJ, Giardiello FM, Johnson DA, Levin TR. Guidelines for Colonoscopy Surveillance After Screening and Polypectomy: A Consensus Update by the US Multi-Society Task Force on Colorectal Cancer. Gastroenterology. 2012;143(3):844-57. https://doi.org/10.1053/j.gastro.2012.06.001

16. Chokshi RV, Hovis CE, Hollander T, Early DS, Wang JS. Prevalence of missed adenomas in patients with inadequate bowel preparation on screening colonoscopy. Gastrointest Endosc. 2012;75(6):1197-203. https://doi.org/10.1016/j.gie.2012.01.005

17. Krajicek E, Fischer M, Allegretti JR, Kelly CR. Nuts and Bolts of Fecal Microbiota Transplantation. Clin Gastroenterol Hepatol. 2019;17(2):345-52. https://doi.org/10.1016/j.cgh.2018.09.029

18. Rutherford CC, Calderwood AH. Update on Bowel Preparation for Colonoscopy. Curr Treat Options Gastroenterol. 2018;16(1):165-81. https://doi.org/10.1007/s11938-018-0165-3

19. Chaussade S, Schmöcker C, Toulemonde P, MuñozNavas M, O’Mahony V, Henri F. Phosphate tablets or polyethylene glycol for preparation to colonoscopy? A multicentre non-inferiority randomized controlled trial. Surg Endosc. 2017;31(5):2166-73. https://doi.org/10.1007/s00464-016-5214-1

20. Munsterman ID, Cleeren E, van der Ploeg T, Brohet R, van der Hulst R. 'Pico-Bello-Klean study': effectiveness and patient tolerability of bowel preparation agents sodium picosulphate-magnesium citrate and polyethylene glycol before colonoscopy. A single-blinded randomized trial. Eur J Gastroenterol Hepatol. 2015;27(1):29-38. https://doi.org/10.1097/MEG.0000000000000192

21. Gweon T-G, Kim SW, Noh Y-S, Hwang S, Kim N-Y, Lee Y, Lee SW, Lee SW, Lee JY, Lim CH, Hun Kim H, Kim JS, Kyung Cho Y, Myung Park J, Seok Lee I, Myung-Gyu Choi. Prospective, Randomized Comparison of SameDay Dose of 2 Different Bowel Cleanser for Afternoon Colonoscopy: Picosulfate, Magnesium Oxide, and Citric Acid Versus Polyethylene Glycol. Medicine (Baltimore). 2015;94(13):e628. https://doi.org/10.1097/MD.0000000000000628

22. Jeon SR, Kim HG, Lee JS, Kim J-O, Lee TH, Cho J-H, Kim $\mathrm{YH}, \mathrm{Cho} J Y$, Lee JS. Randomized controlled trial of lowvolume bowel preparation agents for colonic bowel preparation: 2-L polyethylene glycol with ascorbic acid versus sodium picosulfate with magnesium citrate. Int J Colorectal Dis. 2015;30(2):251-8. https://doi.org/10.1007/s00384-014-2066-9 
23. Martel M, Barkun AN, Menard C, Restellini S, Kherad O, Vanasse A. Split-Dose Preparations Are Superior to Day-Before Bowel Cleansing Regimens: A Meta-analysis. Gastroenterology. 2015;149(1):79-88. https://doi.org/10.1053/j.gastro.2015.04.004

24. Hosoe N, Nakashita M, Imaeda H, Sujino T, Bessho R, Ichikawa R, Inoue N, Kanai T, Hibi T, Ogata H. Comparison of patient acceptance of sodium phosphate versus polyethylene glycol plus sodium picosulfate for colon cleansing in Japanese: Patient acceptance for laxative. J Gastroenterol Hepatol. 2012;27(10):1617-22. https://doi.org/10.1111/j.1440-1746.2012.07190.x

25. Wexner SD, Force T, Beck DE, Baron TH, Fanelli RD, Hyman N, Wasco KE; American Society of Colon and Rectal Surgeons; American Society for Gastrointestinal Endoscopy; Society of American Gastrointestinal and Endoscopic Surgeons. A consensus document on bowel preparation before colonoscopy: Prepared by a Task Force From The American Society of Colon and Rectal Surgeons (ASCRS), the American Society for Gastrointestinal Endoscopy (ASGE), and the Society of American Gastrointestinal and Endoscopic Surgeons (SAGES). Gastrointest Endosc. 2006;63(7):894-909. https://doi.org/10.1016/j.gie.2006.03.918

26. Landreneau SW, Di Palma JA. Update on Preparation for Colonoscopy. Curr Gastroenterol Rep. 2010;12(5):366-73. https://doi.org/10.1007/s11894-010-0121-4

27. Weir MA, Fleet JL, Vinden C, Shariff SZ, Liu K, Song H, Jain AK, Gandhi S, Clark WF, Garg AX. Hyponatremia and Sodium Picosulfate Bowel Preparations in Older Adults. Am J Gastroenterol. 2014;109(5):686-94. https://doi.org/10.1038/ajg.2014.20

28. Gandhi S, Shariff SZ, Fleet JL, Weir MA, Jain AK, Garg AX. Validity of the International Classification of Diseases 10th revision code for hospitalisation with hyponatraemia in elderly patients. BMJ Open. 2012;2(6):e001727. https://doi.org/10.1136/bmjopen-2012-001727

29. Calderwood AH, Jacobson BC. Comprehensive validation of the Boston Bowel Preparation Scale. Gastrointest Endosc. 2010;72(4):686-92. https://doi.org/10.1016/j.gie.2010.06.068

30. Chaves Marques S. The Boston Bowel Preparation Scale: Is It Already Being Used? GE - Port J Gastroenterol. 2018;25(5):219-21. https://doi.org/10.1159/000486805
31. Massinha P, Almeida N, Cunha I, Tomé L. Clinical Practice Impact of the Boston Bowel Preparation Scale in a European Country. GE - Port J Gastroenterol. 2018;25(5):230-5. https://doi.org/10.1159/000485567

32. Declaración de Helsinki de la AMM - Principios éticos para las investigaciones médicas en seres humanos. Asociación Médica Mundial; 2017.

33. Resolución número 8430 de 1993. Por la cual se establecen las normas científicas, técnicas y administrativas para la investigación en salud. Ministerio de Salud (4 de octubre de 1993).

34. Resolución No. 2018013725 DE 5 de abril de 2018. Por la cual se concede un Registro Sanitario. Ministerio de Salud, INVIMA (5 de abril de 2018).

35. Lai EJ, Calderwood AH, Doros G, Fix OK, Jacobson BC. The Boston bowel preparation scale: a valid and reliable instrument for colonoscopy-oriented research. Gastrointest Endosc. 2009;69(3):620-5. https://doi.org/10.1016/j.gie.2008.05.057

36. Sullivan KM, Dean AG. OPENEPI [Internet] [acceso el 15 de junio de 2019]. Disponible en: https://www.openepi. com/SampleSize/SSCohort.htm

37. Gu P, Lew D, Oh SJ, Vipani A, Ko J, Hsu K, Mirakhor E, Pattisapu V, Bullen T, Fuller G, Spiegel BMR, Almario CV. Comparing the Real-World Effectiveness of Competing Colonoscopy Preparations: Results of a Prospective Trial. Am J Gastroenterol. 2019;114(2):305-14. https://doi.org/10.14309/ajg.0000000000000057

38. Kim ES, Lee WJ, Jeen YT, Choi HS, Keum B, Seo YS, Chun HJ, Lee HS, Um SH, Kim CD, Ryu HS.A randomized, endoscopist-blinded, prospective trial to compare the preference and efficacy of four bowel-cleansing regimens for colonoscopy. Scand J Gastroenterol. 2014;49(7):871-7. https://doi.org/10.3109/00365521.2014.910543

39. Basch E, Schrag D. The Evolving Uses of "Real-World" Data. JAMA. 2019;321(14):1359-1360. https://doi. org/10.1001/jama.2019.4064

40. Kim J, Kim HG, Kim KO, Kim HW, Park J, Byeon JS, Hwang SW, Shin HD, Shin JE, Yang HJ, Lee HS, Jung Y, Cho YS, Joo YE, Myung DS, Huh KC, Ahn EM. Clinical comparison of low-volume agents (oral sulfate solution and sodium picosulfate with magnesium citrate) for bowel preparation: the EASE study. ntest Res. 2019;17(3):413-418. https://doi.org/10.5217/ir.2018.00156 